\title{
LA COBERTURA EDUCATIVA EN ESCUELAS PÚBLICAS DEL DEPARTAMENTO DE BOLÍVAR (COLOMBIA): UN ANÁLISIS DE EFICIENCIA Y PRODUCTIVIDAD
}

Francisco Maza-Avila Universidad de Cartagena Colombia

Ivan Batista-Ochoa Universidad Tecnologica de Bolivar Colombia

Juan Carlos Vergara-Schmalbach Universidad de Cartagena Colombia 
Panorama Económico, Vol. 25 - No. 4 (Octubre - Diciembre de 2017), pp. 559-576

Francisco Maza-Avila

Ivan Batista-Ochoa

Juan Carlos Vergara-Schmalbach

\title{
La cobertura educativa en escuelas públicas del departamento de Bolívar (Colombia): Un análisis de eficiencia y productividad
}

\section{Resumen \\ Este artículo analiza la eficiencia y la productividad en la cobertura de las escuelas públicas de 44 municipios del departamento de Bolívar, Colombia, para el periodo 2011-2013, empleando para ello el Análisis Envolvente de Datos y el Índice de Productividad de Malmquist, a partir de la información suministrada por el Ministerio de Educación Nacional de Colombia y la Secretaria de Educación del departamento de Bolívar, relacionada con el número de estudiantes matriculados, número de docentes, inversión y los metros cuadrados por establecimiento educativo. Los resultados indican una desmejora de la eficiencia técnica de todos los establecimientos educativos en el periodo de análisis. Con respecto a la productividad, se observa una disminución durante el periodo considerado, pese a la evolución positiva de la tecnología. Se pude concluir, que los municipios no están utilizando de forma eficiente y productiva los recursos públicos destinados para mejorar la cobertura educativa. \\ Palabras clave: Eficiencia, productividad, cobertura educativa, Análisis Envolvente de Datos, Indice de Malmquist. \\ Clasificación JEL: H52, I20, I22, I28 \\ Coverage of education in public schools of the Bolivar Region (Colombia). An analysis of eficiency and productivity}

\begin{abstract}
This research analyze the efficiency and productivity of educational coverage of public schools in 44 municipalities of Bolivar region in Colombia for the years 2011-2013, through Data Envelopment Analysis (DEA) and Malmquist Index. Data provided by the Ministry of Education and the Secretary of Education of the Bolivar region considers the number o students, professors, investment levels, and infraestructure (measured in m2). Results indicate that technical efficiency deteriorated in all units, and productivity worsens despite technological upgrades. By these, municipalities are not using efficiently all the resources oriented to increase educational coverage.

Keywords: Efficiency, Productivity, Educational Coverage, Data Envelopment Analysis, Malmquist Index. JEL Classification: H52, I20, I22, I28
\end{abstract}

\section{Couverture de l'enseignement dans les écoles publiques de la région de Bolivar (Colombie). Une analyse d'efficacité et de productivité}

\footnotetext{
Résumé

Cette recherche analyse l'efficacité et la productivité de la couverture éducative des écoles publiques dans 44 municipalités de la région de Bolivar en Colombie pour la période 2011-2013, par le biais de l'analyse du développement de données (DEA) et de l'indice de Malmquist. Les données fournies par le ministère de l'Éducation et le secrétaire à l'Éducation de la région de Bolivar prennent en compte le nombre d'étudiants, de professeurs, les niveaux d'investissement et les infrastructures (mesurés en $\mathrm{m} 2$ ). Les résultats indiquent que l'efficacité technique s'est détériorée dans toutes les unités et que la productivité s'est détériorée malgré les améliorations technologiques. Ainsi, les municipalités n'utilisent pas efficacement toutes les ressources destinées à accroître la couverture éducative.

Mots-clés: Efficacité, productivité, couverture éducative, analyse de l'enveloppement des données, Indice de Malmquist.
}

Nomenclature JEL: H52, I20, I22, I28 


\section{La cobertura educativa en escuelas públicas del departamento de Bolívar (Colombia): Un análisis de eficiencia y productividad}

INFORMACIÓN DEL ARTÍCULO

Recepción de artículo: 11/04/2017

Concepto de evaluación: 19/05/2017

Aceptación de artículo: 27/06/2017
Francisco Maza-Avila* Universidad de Cartagena, Colombia

Ivan Batista-Ochoa Universidad Tecnologica de Bolivar, Colombia

Juan Carlos Vergara-Schmalbach Universidad de Cartagena, Colombia

\section{INTRODUCCIÓN}

En Colombia, la educación está establecida en el artículo $N^{\circ} 67$ de la Constitución Política de Colombia de 1991 como un derecho de la persona y un servicio público con función social y de completa gratuidad en instituciones del Estado. Ésta se encuentra ampliamente financiada por el Sistema General de Participaciones -SGP-, cuyo objetivo consiste en transferir los recursos del Estado -recaudados por concepto de impuestos, regalías, multas entre otrosa los entes territoriales ${ }^{1}$ para satisfacer las necesidades en la prestación de los servicios de educación, salud, agua potable y saneamiento básico, así como aquellos denominados de propósito general. Para el caso de la educación, el SGP financia las acciones encaminadas a garantizar tanto la cobertura como la calidad de la educación de los establecimientos educativos de los municipios de Colombia. Este sistema cuenta con un control por parte del Departamento de planeación Nacional -DNP-, quien realiza actividades de ejecución, monitoreo, seguimiento a los recursos del SGP, procurando su asignación eficiente (Quesada, Blanco, \& Maza, 2010). Sin embargo, entre sus debilidades actuales se encuentra el control sobre el uso correcto y eficiente de los recursos por parte de las entidades territoriales, lo que ha derivado, entre otras cosas, a problemas en cuanto a cobertura y calidad educativa. Para el caso del departamento de Bolívar el panorama ha sido dramático, pues en el 2010 fue categorizado con riesgo crítico-alto en educación, es decir, que las entidades estaban poniendo en riesgo el cumplimiento de las metas de calidad, cobertura y continuidad en la prestación de los servicios por hacer un uso inadecuado de los recursos.

\footnotetext{
* Autor para correspondencia

Correos electrónicos: fmazaa@unicartagena.edu.co*, batistaochoaivan@gmail.com, jvergaras@unicartagena.edu.co 1 Departamentos, municipios y distritos, dentro de los cuales se encuentra Bolívar como departamento y Cartagena como Distrito turístico y cultural (Ministerio de Hacienda y Crédito Público, 2013).
} 
Por lo anterior, y dada la importancia de la educación por su gran aporte al desarrollo socialdelosterritorios, estaartículoanalizala eficiencia y la productividad en la cobertura delas escuelas públicas deeducación básica y media de 44 municipios -que corresponden a los no certificados en educación ${ }^{2}$ - del departamento de Bolívar para el período 2011-2013. Para ello, este artículo se ha estructurado de la forma siguiente: en la primera sección se muestra como está organizada la educación en Colombia a la luz de la constitución y las leyes que la regulan; en la siguiente sección se revisan las metodologías para el análisis de la eficiencia y de la productividad -Análisis Envolvente de Datos e Índice de Productividad de Malmquist-; luego se presenta la función de producción del proceso de cobertura de la educación básica y media del departamento de Bolívar y los datos utilizados para el análisis; seguidamente se aplican las metodologías Análisis Envolvente de Datos e Índice de Malmquist para la medición de la eficiencia y de la productividad a partir de la información suministrada por el por el Ministerio de Educación Nacional de Colombia y la Secretaria de Educación del departamento de Bolívar; por último, se presenta el análisis de los resultados y las conclusiones.

\section{La educación en Colombia}

En Colombia, la educación se define como un proceso de formación permanente, personal, cultural y social que se fundamenta en una concepción integral de la persona humana, de su dignidad,

\footnotetext{
2 Los municipios certificados asumen la competencia en virtud de la certificación, y son plenamente responsables del manejo integral de la educación en su entidad territorial, una vez suscriban las correspondientes actas de entrega y recibo de los recursos (físicos, financieros, humanos y técnicos) destinados al sector. Los municipios no certificados asumen la competencia sin virtud de la certificación, y no son responsables del manejo integral de la educación en su entidad territorial.
}

de sus derechos y de sus deberes. La Constitución Política de Colombia establece las bases fundamentales de la naturaleza del servicio educativo. Si bien en la carta política la educación no está consignada bajo el acápite de los derechos fundamentales (Quesada Ibargüen, Blanco Hernández, \& Maza Avila, 2010), en el artículo 67 se explica que se trata de un derecho de la persona, de un servicio público que tiene una función social y que corresponde al Estado regular y ejercer la suprema inspección y vigilancia respecto a este servicio (MEN, 2010).

La estructura del sistema educativo en Colombia se encuentra establecida en el Título II de la Ley 115 de 1994, por la cual se expide la Ley General de Educación, y debe ser acatada por las instituciones educativas del Estado y por los establecimientos educativos fundados por particulares bajo las normas pertinentes y la reglamentación del Gobierno Nacional. Inicialmente, se encuentra la Educación Formal (Figura 1), que impartida en establecimientos educativos aprobados en una secuencia regular de ciclos lectivos, con sujeción a pautas curriculares y progresivas, y conducente a grados y títulos $\mathrm{y}$, por otro lado, se encuentra la Educación No Formal, término sustituido por "Educación para el trabajo y el desarrollo humano" mediante la Ley 1064 de 2006 y que pretende dotar de competencias necesarias para satisfacer las necesidades del mercado laboral, dando lugar a la obtención de un certificado de aptitud ocupacional (MEN, 2009a). En este trabajo está enfocado, especialmente, en la Educación Formal, en particular, la educación básica y media.

Como ya se mencionó con anterioridad, la educación oficial, al igual que otros sectores, es financiada por el Estado, 
Figura 1. Estructura de la Educación Formal en Colombia

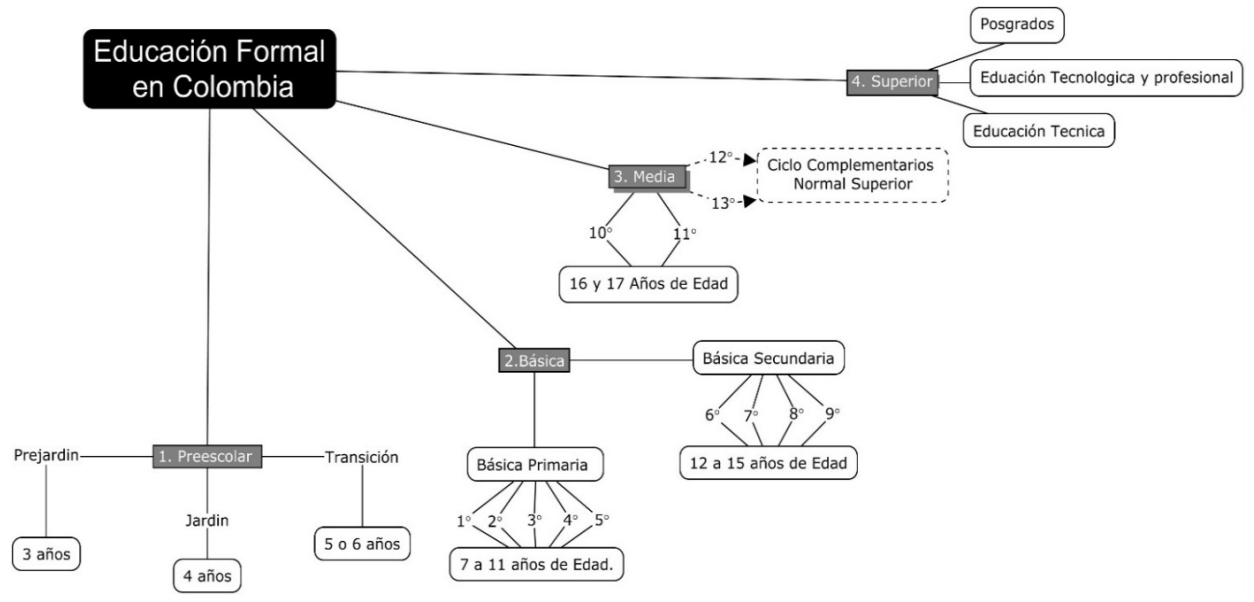

Fuente: Elaboración propia, en base a la Guía N³3 del MEN: Organización del sistema educativo

principalmente, a través del SGP, el cual corresponde a los recursos que la Nación debe transferir a las entidades territoriales -departamentos, distritos y municipios- en cumplimiento de los artículos 356 y 357 de la Constitución Política reformados por los Actos Legislativos 01 de 2001 y 04 de 2007 (MEN, 2009b). Teniendo en cuenta los criterios de racionalidad del gasto público, es de gran importancia para el Estado determinar qué tan eficiente y productiva es la inversión de los recursos destinados a este sector, de allí que el Departamento de Planeación Nacional -DNP-, brinde las pautas a los departamentos y municipios parala presentación deinformesperiódicos de eficiencia en calidad y cobertura educativa en la aplicación de recursos del Sistema General de Participaciones -SGP-, (Maza Avila, Quesada Ibargüen, \& Vergara Schmalbach, 2013; Quesada Ibargüen et al., 2010) sugiriendo el uso de la metodología DEA para la evaluación de la eficiencia.

Modelo para el análisis de la eficiencia: el Análisis Envolvente de Datos

El análisis envolvente de datos -DEA-, es una técnica no paramétrica utilizada para medir la eficiencia de unidades de toma de decisión -o DMU. Por sus siglas en inglés- y fue propuesta por Charnes en las Ciencias de la Administración en 1978 (Charnes, Cooper, \& Rhodes, 1978). Se considera que cada DMU está involucrada en un proceso de transformación, en el cual consumiendo una cantidad de inputs -recursos- intenta producir outputs -bienes o servicios-; por tanto, los modelos DEA parten de las cantidades de inputs empleadas y los outputs producidos por un grupo de DMU, donde se combinan las mejores características de dos o más productores para la construcción de una frontera de eficiencia (Sanchez, Parra, \& Udi, 2014). En esta metodología, la formulación del modelo puede estar orientada a la producción de mayores outputs o a la reducción de Inputs. El modelo orientado a los Inputs busca la minimización de los insumos para la producción de un nivel dado de producto. Por su parte, el modelo orientado a los Outputs busca la maximización de la producción dadas unas cantidades de insumos. En otras palabras, el primer modelo provee una medida sobre qué tanto están siendo subutilizados los insumos, mientras que 
el segundo se preocupa por medir qué tanto se podría llegar a producir con esos insumos (Gamarra, 2004).

Según lo planteado por Maza, Quesada y Vergara (2013), el modelo se formaliza asumiendoque hay $n$ DMU a ser evaluadas, cada una de las cuales consumen $m$ inputs diferentes para producir $s$ outputs también diferentes. La DMUj utiliza un monto de $\quad X_{i}=x_{i j}$ inputs $(i=1, \ldots, m)$ $\mathrm{y}$ produce un monto de $Y_{j}=Y_{k j}$ productos $(k=1, \ldots, s)$ La matriz de medida del producto es designada por, y la de medida de los inputs se designa por $\mathrm{X}$. Se asume además que $\geq 0 \mathrm{y} \geq 0$. Asimismo, se permiten rendimientos constantes -por ejemplo, el modelo de Charnes, Cooper y Rhodes, CCR- o variables a escala -modelo de Banker, Charnes y Cooper, BCC- (Quesada Ibargüen et al., 2010).

El primer modelo -CCR-, fue desarrollado por Charnes, Cooper y Rhodes quienes publicaron su hallazgo en The European Journal of Operational Research y seutiliza cuando el proceso presenta rendimientos constantes a escala, es decir, un incremento en los inputs produce un incremento proporcional en los outputs (Charnes et al., 1978). En este modelo se considera la eficiencia de una DMU como la razón entre la producción ponderada total y el consumo ponderado total, el valor de estas ponderaciones las proporciona el modelo sin necesidad de conocer los precios de las entradas o las salidas. El segundo modelo -BCC- cuyas siglas obedecen a las iniciales de sus autores Banker, Charnes y Cooper (1984) es utilizado en aquellos casos donde un incremento en los inputs no equivale a un incremento proporcional en los outputs. Lo que busca este modelo es comparar cada DMU con aquellas que sean equivalentes en tamaño, y no con todas las que se encuentren en el problema (Cavadia \& Maldonado, 2009). El modelo CCR con orientación input se sigue por la siguiente fórmula:

$$
\begin{aligned}
& \text { MAX } \sum_{k=1}^{s} u_{k 0} y_{k 0}=h_{0} \\
& s . a: \sum_{k=1}^{s} u_{k j}{ }_{k j}-\sum_{i=1}^{m} v_{i j} x_{i j} \leq 0 \quad \forall j \\
& \sum_{i=1}^{m} v_{i 0} x_{i 0}=1 \\
& v_{k j} \geq \varepsilon>0 \quad \forall k, j \\
& u_{i j} \geq \varepsilon>0 \quad \forall i, j
\end{aligned}
$$

Donde $v_{i j \text { y }} u_{k j}$ son respectivamente los pesos correspondientes a cada entrada $y$ cada salida; $m$ el número total de entradas consideradas y s el número de salidas de la DMU; $\boldsymbol{\varepsilon}$ es un número infinitesimal -no arquimediano-, que garantiza que ninguna DMU asignará valor cero como peso de algún input u output. Este modelo presentado corresponde a la forma dual, también conocida como la forma envolvente del modelo CCR-Input, que es preferida a la forma primal dado que el método primal toma más tiempo para su resolución por las $n+m+s$ restricciones que tiene mientras que el dual tiene solo $\mathrm{m}+\mathrm{s}$ restricciones (Quesada Ibargüen et al., 2010). La otra dirección que se considera es la orientada a los outputs; aplicada al CCR produce el modelo CCR-output, cuyo modelo es el siguiente:

$$
\begin{aligned}
& M A X \gamma_{0}+\varepsilon\left\lfloor\sum_{k=1}^{s} h_{k}^{+}+\sum_{i=1}^{m} h_{i}^{+}\right\rfloor \\
& s . a: \sum_{k=1}^{s} x_{i j} \delta_{j}=x_{i 0}-h_{i}^{-} \quad \forall i
\end{aligned}
$$




$$
\begin{aligned}
& \text { s. } a: \sum_{j=1}^{n} y_{k j} \delta_{j}=y_{k 0} \gamma_{o}+h_{k}^{+} \forall k \\
& \delta_{j}, h_{i}^{-}, h_{k}^{+} \geq 0 \quad \forall j, i, k \\
& \gamma_{o} \text { irrestricta }
\end{aligned}
$$

Cuando no es procedente asumir una tecnología con retornos a escala constante, por la naturaleza misma del problema o porque se tenga duda respecto a cuál tecnología asumir como supuesto, es recomendable trabajar con retornos a escala variable -VRS por sus siglas del inglés, o BCC por Banker, Charnes y Cooper, los autores del modelo-. Este modelo se logra introduciendo en elCCR en forma envolvente la restricción adicional $\sum_{j=1}^{n} \delta_{j}=1$ eficiencia así calculada se conoce como eficiencia técnica -ET- (Maza Avila et al., 2013; Quesada Ibargüen et al., 2010).

Medición del cambio de la eficiencia y del cambio técnico: el Índice de Productividad de Malmquist

El Índice de Productividad Malmquist -o, simplemente, Índice de Malmquistpermite descomponer el crecimiento de la productividad en dos componentes: cambios en la eficiencia técnica y cambios en la tecnología a lo largo del tiempo (Pérez González, Maza Ávila, Blanco Canto, \& Jiménez García, 2016). Mide el cambio en la productividad entre dos períodos - $\mathrm{t}$ y $\mathrm{t}+1-\mathrm{a}$ partir del cálculo de funciones de distancia con respecto a los dos períodos en estudio, utilizando como referencia la tecnología existente en uno de los mismos. Este índice fue inicialmente introducido en la literatura económica, más específicamente en el ámbito de la teoría del consumo por Sten Malmquist (1953). Posteriormente Caves, Christensen y Diewert (1982) aplicaron por primera vez este índice a la medición de la productividad en contextos de funciones de producción. Luego Färe, Grosskopf, Lindgren y Roos (1989) se dieron a la tarea de aplicarlo en un contexto no paramétrico, pero finalmente fueron Färe, Grosskopf, Norris y Zhang (1994) quienes popularizaron el modelo.

El Índice de Productividad de Malmquist ha sido utilizado por diferentes autores en múltiples estudios y enfocados a distintos sectores, entre ellos: Hjalmarsson \& Veiderpass (1992), Bjurek \& Hjalmarsson (1995), Grifell-Tatjé \& Lovell (1995), Liu \& Wang (2008), Asmild, Paradi, Aggarwall, \& Schaffnit (2004), Kumar (2006), Coelli \& Rao (2005). Vale anotar que existen distintos tipos de índices para el análisis del cambio en la productividad, siendo los más populares el Índice de Törnqvist, el Índice de Fisher y el Índice de Malmquist. La ventaja de este último radica en que no se requiere disponer tanto de las cantidades como de los precios de todos los inputs y los outputs, tal como lo exigen los dos primeros (Marcelino Martinez, 2002).

Siguiendo la metodología propuesta por Färe, Grosskopf, Norris y Zhang (1994), la definición del Índice de Malmquist basado en el output supone que en cada período $t=1, \ldots T$, la tecnología en producción $S^{t}$ modela la transformación de inputs, $X^{t} \in \mathfrak{R}^{N}$ en outputs, $Y^{t} \in \mathfrak{R}^{M}$.

$S^{t}=\left\{\left(X^{t}, Y^{t}\right): X^{t}\right.$ puede producir $\left.Y^{t}\right\}$

Para elaborar el Índice de Malmquist es preciso definir funciones de distancia con respecto a dos períodos diferentes. La función de distancia del output en $t$ se especifica como:

$$
\begin{aligned}
& \left(X^{t}, Y^{t}\right)=\inf \left\{\varnothing:\left(X^{t}, Y^{t} / \varnothing\right) \in S^{t}\right\} \\
& =\left(\sup \left\{\emptyset:\left(X^{t}, \emptyset Y^{t}\right) \in S^{t}\right\}\right)
\end{aligned}
$$


Esta función se define como el recíproco de la máxima expansión proporcional del vector de output $Y^{t}$, dados los inputs $X^{t}$, y caracteriza completamente la tecnología. En particular, $D_{0}^{t}\left(X^{t}, Y^{t}\right) \leq 1$ si $\mathrm{y}$ solo si : $\left(X^{t}, Y^{t}\right) \in S^{t}$. Adicionalmente, $D_{0}^{t}\left(X^{t}, Y^{t}\right)=1$ si y solo si $\left(X^{t}, Y^{t}\right)$ está en la frontera tecnológica. En la terminología de Farell (1957) este último caso ocurre cuando la producción es técnicamente eficiente. La función de distancia anterior mide el máximo cambio proporcional en outputs requerido para conseguir que $\left(X^{t+1}, Y^{t+1}\right)$ sea factible en relación con la tecnología en $t$. De forma similar, se puede definir la función de distancia que mida la máxima proporción de cambio en output necesaria para que la combinación $\left(X^{t}, Y^{t}\right)$ sea factible con relación a la tecnología en $t+1$, que se denomina $D_{0}^{t+1}\left(X^{t}, Y^{t}\right)$. Así pues el índice de productividad en output de Malmquist se define como:

$$
M^{t}=\frac{D_{0}^{t}\left(X^{t+1}, Y^{t+1}\right)}{D_{0}^{t}\left(X^{t}, Y^{t}\right)}
$$

Donde la tecnología en $t$ es la tecnología de referencia. Alternativamente, es posible definir un índice de Malmquist basado en el período $t+1$ :

$$
M^{t+1}=\frac{D_{0}^{t+1}\left(X^{t+1}, Y^{t+1}\right)}{D_{0}^{t+1}\left(X^{t}, Y^{t}\right)}
$$

La elección de una u otra tecnología de referencia resulta una cuestión relevante. Por este motivo, para resolver el problema que puede representar la consideración de una tecnología fija, Fare et al. (1994) definen el Índice de Malmquist de cambio en productividad basado en el output como la media geométrica de los Índices de Malmquist especificados con anterioridad:

$$
M_{0}\left(X^{t+1}, Y^{t+1}, X^{t}, Y^{t}\right)=\left[\left(\frac{D_{0}^{t}\left(X^{t+1}, Y^{t+1}\right)}{D_{0}^{t}\left(X^{t}, Y^{t}\right)}\right)\left(\frac{D_{0}^{t+1}\left(X^{t+1}, Y^{t+1}\right)}{D_{0}^{t+1}\left(X^{t}, Y^{t}\right)}\right)\right]^{1 / 2}
$$

O, en forma equivalente:

$$
M_{0}\left(X^{t+1}, Y^{t+1}, X^{t}, Y^{t}\right)=\left(\frac{D_{0}^{t+1}\left(X^{t+1}, Y^{t+1}\right)}{D_{0}^{t}\left(X^{t}, Y^{t}\right)}\right) \times\left[\left(\frac{D_{0}^{t}\left(X^{t+1}, Y^{t+1}\right)}{D_{0}^{t+1}\left(X^{t+1}, Y^{t+1}\right)}\right)\left(\frac{D_{0}^{t}\left(X^{t}, Y^{t}\right)}{D_{0}^{t+1}\left(X^{t}, Y^{t}\right)}\right)\right]^{1 / 2}
$$

Esta última expresión es la que permite desagregar la evolución que sigue la productividad en los dos componentes antes mencionados. En la primera parte de la ecuación, se hace referencia al cambio en la eficiencia técnica de las DMU'S -CE-, cuyas mejoras se consideran evidencia de "catching-up", es decir, de acercamiento de cada una de las DMU a la frontera eficiente. Por su parte, el segundo componente de la ecuación indica cómo varía el cambio técnico y se conoce como "índice de productividad de frontera" -CT-, y muestra la distancia relativa entre las fronteras y por tanto, si el desplazamiento de la frontera eficiente hacia el input de cada DMU está generando una innovación en esta última (Martín Bofarull, 2002). Según Coll \& Blasco (2006) para la interpretación de los resultados es necesario conocer que se deben analizar los datos de la siguiente manera:

- $\mathrm{Si} \mathrm{CE}>1 \mathrm{Se}$ ha producido una ganancia en eficiencia, la unidad evaluada se encuentra más cerca de la 
frontera tecnológica de rendimientos constantes en el periodo $t+1$ de lo que estaba en el periodo anterior. Si CE $<1$ La DMU se ha alejado de la frontera, es decir, ha perdido eficiencia y Si CE =1 La unidad evaluada no ha visto modificada su posición con relación a la frontera.

- Si el CT=1 con referencia a la DMU bajo análisis, significa que no ha habido estancamiento de la tecnología, si CT >1 se puede observar que ha habido un progreso tecnológico y si CT $<1$ quiere decir que se ha producido un regreso tecnológico en la frontera.

- Por su parte si $\mathrm{IM}=1$ representa que con referencia a la DMU en estudio se puede decir que durante los periodos estudiados se ha mantenido la productividad- producir el mismo nivel de salidas con las mismas entradas-, si IM $>1$ significa que la DMU aumento su productividad $y$ por el contrario si la IM<1 se puede observar que la DMU ha rebajado la productividad.

\section{METODOLOGÍA}

La utilización de la metodología Análisis Envolvente de Datos para la medición de la eficiencia en la educación, ha sido abordada por diferentes autores y desde distintas perspectivas, atendiendo al problema de la cobertura, la calidad y la distribución adecuada óptima de los recursos utilizados. Los estudios realizados han sido aplicados tanto al sector privado como al sector público e, incluso, se presentan estudios que involucran a los dos sectores y que, en definitiva, buscan contribuir a los procesos de toma de decisiones. Entre los estudios realizados en este campo se encuentran los realizados por Quesada Ibargüen, Blanco Hernández, \& Maza Avila (2010), Maza Ávila, Quesada
Ibargüen, \& Vergara Schmalbach (2013), Iregui B., Melo F, \& Ramos (2006), Torres Cardemas \& Reynosa Carrero (2011), Restrepo \& Villegas R. (2007), López Gómez \& Rivera Monsalve (2008), Chediak \& Rodriguez (2011), Arenas Valencia, Soto Mejía, \& Marino Rivera (2004), Soto Mejía, Vásquez Artunduaga, \& Villegas Flórez (2009), Barbosa Gómez (2010), Lopez Munoz \& Suarez Baquero (2012). Para el caso de esta investigación, se evalúa para la unidad de decisión -municipios- la cantidad de productos -outputs-obtenidos dado unos niveles de insumo -inputs-, obteniendo resultados que sirven de guía en la toma de decisiones a nivel departamental. El modelo de producción propuesto por el Departamento Nacional de Planeación -DNP- sugiere a los entes territoriales medir la eficiencia en dos productos fundamentales: la matrícula educativa y la calidad de la educación. Esta investigación se refiere al primer producto: la matrícula educativa -cobertura-.

La matrícula educativa puede entenderse como la inclusión, registro o inscripción de la población en el sistema educativo. En este estudio, para la producción de la matrícula se estima la eficiencia a nivel de cada escuela o establecimiento público de cada uno de los 44 municipios del departamento de Bolívar analizados, tomando como objeto de análisis los municipios no certificados, teniendo en cuenta que las dinámicas de la prestación del servicio y las condiciones de asignación de recursos difieren de los municipios certificados. En la Tabla 1, se presentan las variables de insumo y producto que se tuvieron en cuenta para la estimación de la eficiencia y de la productividad en la matricula educativa.

Para la estimación de la eficiencia se utiliza el modelo BCC orientado al Output, 


\section{Tabla 1. Descripción de las variables de la función de producción de cobertura en la educación del departamento de Bolívar.}

\begin{tabular}{|l|l|l|}
\hline \multicolumn{1}{|c|}{ VARIABLE } & \multicolumn{1}{|c|}{ ACLARACIÓN } & \multicolumn{1}{c|}{ FUENTE } \\
\hline $\begin{array}{l}\text { Producto 1: Número } \\
\text { de matriculados por } \\
\text { establecimiento educativo }\end{array}$ & $\begin{array}{l}\text { Total de estudiantes } \\
\text { matriculados en los } \\
\text { establecimientos educativos del } \\
\text { sector oficial (escuelas públicas) } \\
\text { del departamento de Bolívar }\end{array}$ & $\begin{array}{l}\text { Ministerio de Educación } \\
\text { Nacional de Colombia, } \\
\text { Secretaria de Educación del } \\
\text { departamento de Bolívar. }\end{array}$ \\
\hline $\begin{array}{l}\text { Insumo 1: Inversión por } \\
\text { establecimiento educativo }\end{array}$ & $\begin{array}{l}\text { Inversión total hecha por } \\
\text { establecimiento educativo del } \\
\text { sector oficial (escuelas públicas) } \\
\text { del departamento de Bolívar, } \\
\text { en esta inversión no se incluye } \\
\text { el pago de administración }\end{array}$ & $\begin{array}{l}\text { Ministerio de Educación } \\
\text { Nacional de Colombia, } \\
\text { Secretaria de Educación del } \\
\text { departamento de Bolívar. }\end{array}$ \\
\hline $\begin{array}{l}\text { Insumo 2: Número de docentes } \\
\text { por establecimiento educativo }\end{array}$ & $\begin{array}{l}\text { Planta docente completa por } \\
\text { establecimiento educativo del } \\
\text { sector oficial (escuela pública) } \\
\text { en el departamento de Bolívar }\end{array}$ & $\begin{array}{l}\text { Ministerio de Educación } \\
\text { Nacional de Colombia, } \\
\text { Secretaria de Educación del } \\
\text { departamento de Bolívar. }\end{array}$ \\
\hline $\begin{array}{l}\text { Insumo 3: Metros } \\
\text { cuadrados construidos por } \\
\text { establecimiento educativo }\end{array}$ & $\begin{array}{l}\text { Metros cuadrados construidos } \\
\text { por establecimiento educativo } \\
\text { del sector oficial (escuela } \\
\text { pública) del departamento de } \\
\text { Bolívar }\end{array}$ & $\begin{array}{l}\text { Ministerio de Educación } \\
\text { Nacional de Colombia, } \\
\text { Secretaria de Educación del } \\
\text { departamento de Bolívar. }\end{array}$ \\
\hline
\end{tabular}

Fuente: Elaboración propia.

utilizado en esta investigación porque no se tiene certeza del tipo de retorno que tendrá la función de producción, sin embargo, se lo que se pretende es maximizar la salidas -cobertura-, es decir, obtener mejores resultados en cobertura, dado un nivel de insumos suministrados (Maza Avila et al., 2013). Se evalúan los establecimientos de educación oficiales - o escuela públicas- de los municipios no certificados adscritos al departamento de Bolívar, lo que deja por fuera de este estudio los establecimientos educativos de los municipios de Cartagena y Magangué, por ser municipios certificados. La información de cada municipio fue obtenida de los datos consolidados y validados por el Ministerio de Educación Nacional y la Secretaría de Educación del Departamento de Bolívar, entes públicos encargados de la compilación, del registro, del manejo y del aseguramiento de la información de los departamentos y municipios de Colombia.

Dado que es posible encontrar falta de información para alguna de las unidades evaluadas en cualquiera de los años de estudio en el consolidado de los datos suministrados por el Ministerio de Educación Nacional y la Secretaría de Educación del Departamento de Bolívar, y siendo la propuesta de Maza, Vergara \& Navarro (2012), aquellos municipios que no reportaron información en, al menos, una de las variables que conforman la función de producción se le asignó el valor de cero (0) en la eficiencia final, a modo de "penalización" dentro del modelo por no permitir el cálculo del índice de eficiencia, lo que no significa que esta sea su eficiencia real. Los municipios con establecimientos educativos en esta situación son 6 para todos los años de estudio. 
Para la medición de los cambios en la productividad se utiliza el Índice de Productividad de Malmquist. Para realizar este cálculo correctamente es necesario la disponibilidad de la información para las mismas DMU's durante la totalidad del periodo analizado, debido a que mide el cambio de la evolución de la productividad total de los factores en el tiempo. Por lo anterior, no se contará con los 44 municipios no certificados del departamento, sino con 38 municipios para los cuales hay disponible información en, al menos, un establecimiento educativo.

Tabla 2. Resumen estadístico de variables año 2011, 2012 y 2013

\begin{tabular}{|l|c|c|c|c|c|c|c|c|c|c|c|c|}
\hline \multicolumn{9}{|c|}{2011} & \multicolumn{7}{c|}{2012} & & \multicolumn{1}{c|}{2013} \\
\hline & C & D & M2 & I & C & D & M2 & I & C & D & M2 & I \\
\hline Media & 1259,78 & 39,76 & 1176,06 & $\$ 15.212 .317,48$ & 1220,48 & 39,60 & 1176,06 & $\$ 75.651 .131,78$ & 1131,27 & 45,83 & 1197,39 & $\$ 84.630 .722,69$ \\
\hline Mediana & 1074 & 33 & 566,80 & $\$ 12.502 .448,11$ & 1075 & 33 & 566,80 & $\$ 68.940 .000,00$ & 913 & 39 & 544,83 & $\$ 77.469 .000,00$ \\
\hline $\begin{array}{l}\text { Desviación } \\
\text { estándar }\end{array}$ & 771,92 & 24,77 & 4086,47 & $\$ 9.955 .999,68$ & 771,03 & 24,72 & 4086,47 & $\$ 44.041 .426,44$ & 733,79 & 25,51 & 4252,47 & $\$ 48.296 .072,64$ \\
\hline Rango & 3823 & 133 & 34327,08 & $\$ 42.429 .961,24$ & 3546 & 132 & 34327,08 & $\$ 191.580 .000,00$ & 3474 & 127 & 34327,08 & $217.631 .000,00$ \\
\hline Mínimo & 110 & 5 & 138,03 & $\$ 1.446 .478,53$ & 114 & 6 & 138,03 & $\$ 5.620 .000,00$ & 111 & 6 & 138,03 & $\$ 9.300 .000,00$ \\
\hline Máximo & 3933 & 138 & 34465,10 & $\$ 43.876 .439,77$ & 3660 & 138 & 34465,10 & $\$ 197.200 .000,00$ & 3585 & 133 & 34465,10 & $226.931 .000,00$ \\
\hline
\end{tabular}

Fuente: Cálculos propios, a partir de información suministrada por Secretaría de Educación del Departamento de Bolívar. Convenciones: la letra C hace referencia a la Cobertura Educativa; la letra D hace referencia a los Docentes; M2 hace referencia a los Metros Cuadrados Construidos; y la letra I hace referencia a la Inversión realizada.

\section{RESULTADOS}

\section{Eficiencia técnica para municipios de Bolívar}

Los resultados muestran que los cinco municipios más eficientes para el año 2011 fueron Tiquisio, San Pablo, María La Baja, Villanueva y San Juan Nepomuceno, siendo Tiquisio el municipio que presenta la eficiencia más alta, con un promedio de 93,34\%, Talaigua Nuevo, por su parte, es el municipio que presenta la eficiencia más baja, con un promedio de 64,69\%. Para el año 2012, de los 44 municipios analizados, las eficiencias más altas las presentaron los municipios de Turbaco, Arjona, Marialabaja, Simití y el Carmen de Bolívar; se destaca el municipio de Turbaco, el cual ostenta la eficiencia media más alta con un promedio de 73,93\%, sin embargo, esta eficiencia se encuentra por debajo de la máxima eficiencia alcanzada el año anterior. La eficiencia media más baja para el año 2012 la obtuvo Arenal con un 53,07\%. Ya para el año 2013, se consolidaron los municipios de Tiquisio, San Pablo, Villanueva, San Jacinto y San Juan Nepomuceno, presentando las eficiencias más altas; se destaca el municipio de Tiquisio el cual presenta una eficiencia promedio del 100\%. Cabe anotar que para este año solo un establecimiento educativo de este municipio hizo parte de la evaluación, debido a que fue el único que proporcionó información completa de las variables necesarias para el análisis. El municipio de El Peñón presentó la menor eficiencia media con un promedio de 49,01\% (Tabla 1). 
Se puede establecer también que los municipios más eficientes mencionados anteriormente no se ven directamente influenciados por la cercanía a la capital del departamento que se encuentra ubicada al norte. Por ejemplo, los municipios de Simití y San Pablo que resultaron entre los más eficientes para el año 2011 y 2013, respectivamente, hacen parte del extremo más alejado de la capital. La dispersión de los datos muestra que los municipios con mayores brechas internas fueron Arjona, Mompós y Santa Rosa del Sur, esto se ve influenciado por la cantidad de instituciones que estos municipios tienen; estos municipios tienen el mayor número de instituciones (Tabla 3).

Estos resultados revelan que, en general, los municipios del departamento de Bolívar presentan, en promedio, una eficiencia que podría catalogarse como media, lo que sugiere que no están asignando de forma adecuada los recursos provistos por el Estado para la mejora de la cobertura educativa en cada escuela pública. Por esa razón, es menester de los organismos fiscalizadores mejorar el control en la asignación real de los recursos destinados a la educación, mediante un monitoreo continuo que establezca las oportunidades de mejora en los municipios. Cabe agregar que, aunque sólo el municipio de Tiquisio haya sido eficiente en el año 2013 alcanzando un $100 \%$, durante estos tres años hubo establecimientos educativos que alcanzaron el 100\% de eficiencia. En el 2011 de los 129 establecimientos educativos solo 15 fueron eficientes, y estos se encontraban ubicados en los municipios de Arjona, Córdoba, el Peñon, San Juan Nepomuceno, Simití, Tiquisio, Turbaco, San Pablo y Santa Rosa del Sur. En el 2012 de los 129 establecimientos educativos evaluados, 13 fueron eficientes y en 2013 solo 14 de los 129 evaluados fueron eficientes.

\section{Cambios en la productividad}

El segundo componente del análisis corresponde al cálculo del Índice de Productividad de Malmquist. Tal como se mencionó con anterioridad, para realizar este cálculo correctamente es necesario la disponibilidad de la información para las mismas DMU's durante la totalidad del periodo analizado que, en este caso, corresponden a los años 2011, 2012 y 2013 , debido a que mide el cambio de la evolución de la productividad total de los factores en el tiempo, partiendo de una tecnología común. Por esta razón sólo fueron considerados 38 de los 44 municipios, para los cuales hay disponible información en al menos de un centro educativo.

Los resultados muestran que, de año en año, el nivel de productividad media presenta una mejora que puede ser explicada por el comportamiento de la eficiencia técnica y de los cambios tecnológicos de algunos municipios. Para el período de 2011-2012, se presenta un cambio positivo en el nivel medio de productividad, explicados especialmente por los cambios tecnológicos, pese a que más del $80 \%$ de los municipios presentó un retroceso en el nivel promedio de los cambios en la eficiencia técnica, los cambios tecnológicos que se presentaron fueron significativamente altos, lo que evidencia variaciones positivas en el nivel medio de productividad. Cabe anotar que para este mismo período todos los municipios evaluados presentaron mejoras tecnológicas. De los 38 municipios evaluados, se destacan los municipios de Arenal, Margarita, Mompós, Morales, San Cristobal, San Jacinto del Cauca y San Juan Nepomuceno, presentando un cambio positivo en el nivel medio de productividad del $60,57 \%$. 
Tabla 3. Media de eficiencia -modelo BBC con orientación al Output- por municipios del Departamento de Bolívar. Periodo 2011-2013

\begin{tabular}{|c|c|c|c|c|c|c|c|c|c|c|}
\hline \multirow{2}{*}{ ZODES } & \multirow{2}{*}{ MUNICIPIOS } & \multicolumn{3}{|c|}{2011} & \multicolumn{3}{|c|}{2012} & \multicolumn{3}{|c|}{2013} \\
\hline & & $\mathrm{N}^{\circ}$ EST. & EM & SD & $\mathrm{N}^{\circ}$ EST. & $\mathrm{EM}$ & SD & $\mathrm{N}^{\circ} \mathrm{EST}$. & EM & SD \\
\hline \multirow{6}{*}{$\begin{array}{l}\text { DEPRESIÓN } \\
\text { MOMPOSINA }\end{array}$} & CICUCO & 3 & 0,00 & 0,00 & 3,00 & 0,00 & 0,00 & 3 & 0,00 & 0,00 \\
\hline & HATILLO DE LOBA & 3 & 81,50 & 8,82 & 3,00 & 66,08 & 13,78 & 3 & 55,20 & 21,55 \\
\hline & MARGARITA & 4 & 65,46 & 7,42 & 4,00 & 46,61 & 3,95 & 4 & 61,29 & 4,74 \\
\hline & MOMPÓS & 6 & 78,40 & 10,01 & 6,00 & 62,75 & 10,37 & 6 & 69,53 & 25,49 \\
\hline & SAN FERNANDO & 2 & 79,02 & 0,90 & 2,00 & 50,22 & 5,04 & 2 & 73,69 & 4,13 \\
\hline & TALAIGUA NUEVO & 3 & 64,69 & 5,53 & 3,00 & 54,20 & 6,80 & 3 & 74,45 & 18,39 \\
\hline \multirow{13}{*}{ DIQUE } & ARJONA & 7 & 78,22 & 15,13 & 7,00 & 72,26 & 16,52 & 7 & 81,38 & 14,72 \\
\hline & ARROYOHONDO & 2 & 80,84 & 4,89 & 2,00 & 50,94 & 2,59 & 2 & 64,78 & 8,73 \\
\hline & CALAMAR & 5 & 81,64 & 12,33 & 5,00 & 71,68 & 14,66 & 5 & 77,43 & 11,64 \\
\hline & CLEMENCIA & 2 & 0,00 & 0,00 & 2,00 & 0,00 & 0,00 & 2 & 0,00 & 0,00 \\
\hline & MAHATES & 5 & 73,97 & 9,71 & 5,00 & 63,04 & 13,48 & 5 & 74,84 & 6,11 \\
\hline & SAN CRISTÓBAL & 2 & 65,48 & 4,72 & 2,00 & 57,43 & 13,33 & 2 & 70,26 & 11,49 \\
\hline & SAN ESTANISLAO & 3 & 70,31 & 5,73 & 3,00 & 58,74 & 4,36 & 3 & 77,00 & 8,20 \\
\hline & SANTA CATALINA & 2 & 69,92 & 2,84 & 2,00 & 56,30 & 2,90 & 2 & 70,32 & 10,06 \\
\hline & SANTA ROSA & 2 & 87,11 & 6,70 & 2,00 & 71,73 & 3,21 & 2 & 88,54 & 4,61 \\
\hline & SOPLAVIENTO & 2 & 0,00 & 0,00 & 2,00 & 0,00 & 0,00 & 2 & 0,00 & 0,00 \\
\hline & TURBACO & 8 & 83,72 & 10,87 & 8,00 & 73,93 & 15,64 & 8 & 84,85 & 11,87 \\
\hline & TURBANA & 3 & 78,02 & 5,00 & 3,00 & 61,91 & 7,21 & 3 & 80,61 & 4,98 \\
\hline & VILLANUEVA & 2 & 89,06 & 1,71 & 2,00 & 83,01 & 17,00 & 2 & 93,96 & 6,05 \\
\hline \multirow{7}{*}{ LOBA } & ALTOS DEL ROSARIO & 1 & 77,08 & 0,00 & 1,00 & 66,76 & 0,00 & 1 & 75,00 & 0,00 \\
\hline & BARRANCO DE LOBA & 3 & 85,90 & 10,57 & 3,00 & 77,56 & 9,48 & 3 & 75,23 & 17,68 \\
\hline & EL PEÑON & 4 & 80,46 & 17,08 & 4,00 & 52,89 & 4,89 & 3 & 49,01 & 8,38 \\
\hline & NOROSÍ & 1 & 0,00 & 0,00 & 1,00 & 0,00 & 0,00 & 1 & 0,00 & 0,00 \\
\hline & REGIDOR & 2 & 72,13 & 20,50 & 2,00 & 49,15 & 0,24 & 2 & 78,68 & 4,22 \\
\hline & RIO VIEJO & 2 & 0,00 & 0,00 & 2,00 & 0,00 & 0,00 & 2 & 0,00 & 0,00 \\
\hline & SAN MARTÍN DE LOBA & 3 & 86,80 & 10,48 & 3,00 & 64,83 & 6,12 & 2 & 75,69 & 8,71 \\
\hline \multirow{6}{*}{$\begin{array}{l}\text { MAGDALENA } \\
\text { MEDIO }\end{array}$} & ARENAL & 1 & 66,16 & 0,00 & 1,00 & 53,07 & 0,00 & 1 & 63,39 & 0,00 \\
\hline & CANTAGALLO & 2 & 86,30 & 3,81 & 2,00 & 78,34 & 7,61 & 1 & 85,50 & 0,00 \\
\hline & MORALES & 4 & 82,50 & 9,82 & 4,00 & 57,08 & 7,16 & 3 & 79,91 & 14,50 \\
\hline & SAN PABLO & 3 & 91,22 & 9,11 & 3,00 & 93,76 & 4,46 & 2 & 96,84 & 3,16 \\
\hline & SANTA ROSA DEL SUR & 5 & 84,74 & 10,10 & 5,00 & 74,33 & 16,70 & 4 & 69,62 & 21,38 \\
\hline & SIMITÍ & 6 & 80,81 & 16,90 & 6,00 & 53,69 & 7,72 & 6 & 62,33 & 11,45 \\
\hline \multirow{5}{*}{ MOJANA } & ACHI & 2 & 71,99 & 9,31 & 2,00 & 58,22 & 11,12 & 1 & 70,05 & 8,20 \\
\hline & MONTECRISTO & 2 & 0,00 & 0,00 & 2,00 & 0,00 & 0,00 & 2 & 0,00 & 0,00 \\
\hline & PINILLOS & 3 & 83,55 & 2,63 & 3,00 & 63,29 & 1,31 & 3 & 70,64 & 7,81 \\
\hline & $\begin{array}{l}\text { SAN JACINTO DEL } \\
\text { CAUCA } \\
\end{array}$ & 3 & 75,29 & 19,04 & 3,00 & 61,32 & 8,10 & 2 & 65,08 & 4,60 \\
\hline & TIQUISIO & 3 & 93,34 & 9,41 & 3,00 & 89,12 & 11,17 & 1 & 100,00 & 0,00 \\
\hline \multirow{7}{*}{$\begin{array}{l}\text { MONTES DE } \\
\text { MARÍA }\end{array}$} & CÓRDOBA & 3 & 77,87 & 15,77 & 3,00 & 69,46 & 1,59 & 3 & 81,13 & 14,11 \\
\hline & $\begin{array}{l}\text { EL CARMEN DE } \\
\text { BOLIIVAR } \\
\end{array}$ & 6 & 79,83 & 7,65 & 6,00 & 76,67 & 10,98 & 6 & 84,92 & 16,27 \\
\hline & EL GUAMO & 1 & 68,41 & 0,00 & 1,00 & 57,03 & 0,00 & 1 & 75,41 & 0,00 \\
\hline & MARÍA LA BAJA & 6 & 90,31 & 5,58 & 6,00 & 72,19 & 12,88 & 6 & 62,12 & 17,47 \\
\hline & SAN JACINTO & 2 & 81,04 & 14,88 & 2,00 & 88,99 & 6,76 & 2 & 89,34 & 6,52 \\
\hline & $\begin{array}{l}\text { SAN JUAN } \\
\text { NEPOMUCENO } \\
\end{array}$ & 5 & 87,98 & 10,64 & 5,00 & 67,84 & 13,10 & 5 & 88,82 & 11,12 \\
\hline & ZAMBRANO & 2 & 68,53 & 3,26 & 2,00 & 58,69 & 4,29 & 2 & 64,13 & 4,30 \\
\hline & \multicolumn{2}{|l|}{ EFICIENCIA MEDIA } & 80,07 & 7,25 & & 66,38 & 6,74 & & 74,89 & 8,01 \\
\hline
\end{tabular}

Fuente: Cálculos propios, a partir de información suministrada por Secretaría de Educación del Departamento de Bolívar Convenciones: $\mathrm{N}^{\circ}$ EST. = Número de escuelas oficiales; $\mathrm{EM}=$ Eficiencia Media; $\mathrm{SD}$ = Desviación Estándar

Nota: El valor cero -0- es asignado a los municipios que no reportaron información en alguna de las variables que permita el cálculo de su eficiencia, por lo que es "penalizado", lo que no quiere decir que sea su eficiencia real. 
Por otro lado, municipios como Calamar, el Carmen de Bolívar y María La Baja para este mismo período, presentan establecimientos educativos con IMs inferiores a 1, es decir, establecimientos educativos poco productivos, donde la principal problemática está focalizada en la disminución de la eficiencia, evidenciando que estos establecimientos deben encaminarse a diseñar estrategias para mejorar el uso de los recursos asignados y así aumentar su productividad. Para el período de 2012-2013, se observa un crecimiento en el nivel medio de productividad, impulsado por los cambios significativos en la eficiencia técnica y en la tecnología. Para este mismo período ninguno de los municipios ni de los establecimientos que hicieron parte del análisis obtuvieron un IM por debajo de 1. Sin embargo, se encontraron retrasos tecnológicos en municipios como Achí, Arjona, El Carmen de Bolívar y Mompós (Tabla 5)

\section{CONCLUSIONES}

El presente artículo tuvo como objetivo estimar la eficiencia y los cambios en la productividad en la gestión de los recursos del SGP para garantizar la cobertura educativa en los establecimientos oficiales de educación básica del departamento de Bolívar durante el periodo 2011-2013. Para ello se seleccionaron las variables de entrada -número de docentes, metros cuadrados construidos e inversión, cada una por establecimiento educativo- y la variable de salida que es el número de estudiantes matriculados por cada establecimiento, categorizado en este estudio como cobertura.

Los resultado mostraron que el sector educativo presenta diversas falencias; la eficiencia técnica de los municipios del departamento de Bolívar en general es baja y desmejoró entre el año 2011 y el año 2013, pasando de una eficiencia de $80,07 \%$ a una de $74,89 \%$, se observa que ningún municipio del departamento fue eficiente durante los años de estudio, es decir no tuvieron una eficiencia del 100\%, sin embargo dentro de estos municipios se destacan establecimientos educativos que fueron eficientes y que, a diferencia de los ineficientes, realizaron una distribución estratégica de su planta docente e infraestructura que le permitieron alcanzar mayores niveles de cobertura. Los municipios con mayor eficiencia técnica promedio entre los años 2011 y 2013 fueron Tiquisio y San Pablo, con eficiencias medias superiores al $90 \%$.

En lo que a productividad se refiere, se evidencia una caída en la eficiencia técnica que no fue favorable para la productividad, aunque fue notoria la influencia positiva del cambio tecnológico. Éste análisis refleja que es necesario una mejor asignación y aprovechamiento delos insumos para alcanzar una productividad óptima. Se destacan en la evolución de la productividad los municipios de Arenal, Margarita, Mompós, Morales, San Cristobal, San Jacinto del Cauca y San Juan Nepomuceno que presentaron un cambio positivo en el nivel medio de productividad del 60,57\%. Es importante destacar que los resultados de este trabajo mostraron que los establecimientos educativos eficientes no necesariamente se encontraban cerca de la capital del departamento. Se observó que establecimientos ubicados en Morales y San Pablo, los cuales hacen parte del extremo más alejado de la capital, lograron alcanzar la eficiencia óptima.

Los resultados de esta investigación sugieren la necesidad de que los encargados a nivel departamental y municipal para la distribución de recursos públicos en cada establecimiento educativo lleven a cabo importantes esfuerzos por mejorar 
Tabla 5. Cambio en la eficiencia, cambio técnico e Índice de Malmquist, por municipios del Departamento de Bolívar. Periodo 2011-2013

\begin{tabular}{|c|c|c|c|c|c|c|c|}
\hline ZODES & MUNICIPIOS & \multicolumn{3}{|c|}{ 2011-2012 } & \multicolumn{3}{|c|}{ 2012-2013 } \\
\hline \multirow{5}{*}{$\begin{array}{l}\text { DEPRESIÓN } \\
\text { MOMPOSINA }\end{array}$} & HATILLO DE LOBA & 0,85 & 1,49 & 1,22 & 0,79 & 1,93 & 1,23 \\
\hline & MARGARITA & 0,73 & 2,19 & 1,57 & 1,22 & 1,00 & 1,22 \\
\hline & MOMPÓS & 0,88 & 1,83 & 1,54 & 1,01 & 1,84 & 1,27 \\
\hline & SAN FERNANDO & 0,63 & 2,43 & 1,51 & 1,36 & 1,00 & 1,36 \\
\hline & TALAIGUA NUEVO & 0,86 & 1,76 & 1,52 & 1,28 & 1,06 & 1,34 \\
\hline & MEDIA ZODES DEPRESIÓN MOMPOSINA & 0,81 & 1,91 & 1,49 & 1,11 & 1,45 & 1,27 \\
\hline \multirow{11}{*}{ DIQUE } & ARJONA & 0,96 & 1,52 & 1,44 & 1,09 & 1,13 & 1,22 \\
\hline & ARROYOHONDO & 0,69 & 2,23 & 1,51 & 1,15 & 1,09 & 1,26 \\
\hline & CALAMAR & 0,98 & 1,40 & 1,35 & 1,04 & 1,26 & 1,28 \\
\hline & MAHATES & 0,89 & 1,65 & 1,44 & 1,15 & 1,16 & 1,30 \\
\hline & SAN CRISTÓBAL & 0,94 & 1,71 & 1,53 & 1,19 & 1,14 & 1,35 \\
\hline & SAN ESTANISLAO & 0,89 & 1,66 & 1,46 & 1,21 & 1,08 & 1,31 \\
\hline & SANTA CATALINA & 0,86 & 1,72 & 1,47 & 1,16 & 1,12 & 1,29 \\
\hline & SANTA ROSA & 0,90 & 1,78 & 1,60 & 1,07 & 1,19 & 1,25 \\
\hline & TURBACO & 0,96 & 1,62 & 1,54 & 1,08 & 1,16 & 1,24 \\
\hline & TURBANA & 0,84 & 1,67 & 1,37 & 1,24 & 1,06 & 1,30 \\
\hline & VILLANUEVA & 0,99 & 1,53 & 1,50 & 1,12 & 1,03 & 1,15 \\
\hline & MEDIA ZODES DIQUE & 0,92 & 1,63 & 1,47 & 1,12 & 1,14 & 1,26 \\
\hline \multirow{6}{*}{ ZODES LOBA } & ALTOS DEL ROSARIO & 0,87 & 1,44 & 1,25 & 1,09 & 1,16 & 1,27 \\
\hline & BARRANCO DE LOBA & 1,06 & 1,38 & 1,45 & 0,87 & 1,50 & 1,28 \\
\hline & EL PEÑON & 0,75 & 2,08 & 1,54 & 0,91 & 1,40 & 1,27 \\
\hline & REGIDOR & 0,76 & 1,98 & 1,47 & 1,33 & 0,92 & 1,22 \\
\hline & SAN MARTÍN DE LOBA & 0,89 & 1,65 & 1,47 & 1,06 & 1,24 & 1,31 \\
\hline & MEDIA ZODES LOBA & 0,87 & 1,74 & 1,46 & 1,02 & 1,29 & 1,27 \\
\hline \multirow{6}{*}{$\begin{array}{c}\text { ZODES } \\
\text { MAGDALENA } \\
\text { MEDIO }\end{array}$} & ARENAL & 0,82 & 1,96 & 1,61 & 1,17 & 1,09 & 1,27 \\
\hline & CANTAGALLO & 0,92 & 1,35 & 1,24 & 1,14 & 1,17 & 1,33 \\
\hline & MORALES & 0,79 & 2,03 & 1,58 & 1,20 & 1,04 & 1,24 \\
\hline & SAN PABLO & 0,98 & 1,28 & 1,25 & 1,00 & 1,11 & 1,09 \\
\hline & SANTA ROSA DEL SUR & 0,89 & 1,71 & 1,44 & 0,90 & 1,22 & 1,09 \\
\hline & SIMITÍ & 0,76 & 2,31 & 1,63 & 1,02 & 1,21 & 1,22 \\
\hline & MEDIA ZODES MAGDALENA MEDIO & 0,83 & 1,92 & 1,51 & 1,04 & 1,16 & 1,19 \\
\hline \multirow{4}{*}{$\begin{array}{c}\text { ZODES } \\
\text { MOJANA }\end{array}$} & ACHI & 0,77 & 1,89 & 1,46 & 1,39 & 0,95 & 1,31 \\
\hline & PINILLOS & 0,77 & 1,69 & 1,31 & 1,05 & 1,26 & 1,31 \\
\hline & SAN JACINTTO DEL CAUCA & 0,93 & 1,74 & 1,60 & 1,11 & 1,22 & 1,34 \\
\hline & TIQUISIO & 0,93 & 1,39 & 1,29 & 1,35 & 0,81 & 1,09 \\
\hline & MEDIA ZODES MOJANA & 0,84 & 1,69 & 1,41 & 1,16 & 1,14 & 1,29 \\
\hline \multirow{9}{*}{$\begin{array}{c}\text { ZODES } \\
\text { MONTES DE } \\
\text { MARIA }\end{array}$} & CÓRDOBA & 1,08 & 1,31 & 1,40 & 0,98 & 1,29 & 1,25 \\
\hline & EL CARMEN DE BOLÍVAR & 0,98 & 1,35 & 1,32 & 1,09 & 1,17 & 1,22 \\
\hline & EL GUAMO & 0,84 & 1,83 & 1,53 & 1,18 & 1,10 & 1,30 \\
\hline & MARÍA LA BAJA & 0,84 & 1,43 & 1,18 & 0,88 & 1,80 & 1,33 \\
\hline & SAN JACINTO & 1,28 & 1,24 & 1,59 & 0,84 & 1,45 & 1,22 \\
\hline & SAN JUAN NEPOMUCENO & 0,92 & 1,73 & 1,58 & 1,06 & 1,22 & 1,26 \\
\hline & ZAMBRANO & 0,91 & 1,69 & 1,55 & 1,06 & 1,26 & 1,31 \\
\hline & MEDIA ZODES MONTES DE MARÍA & 0,96 & 1,48 & 1,40 & 1,00 & 1,37 & 1,27 \\
\hline & MEDIA GENERAL & 0,89 & 1,70 & 1,46 & 1,07 & 1,25 & 1,26 \\
\hline
\end{tabular}

Fuente: Elaboración propia. 
su focalización, que estén encaminados a la disminución de las brechas entre los diferentes municipios del departamento de Bolívar en materia de coberturas. También es menester reiterar el llamado a los órganos de control, tal como ya se ha hecho en artículos anteriores (Maza Avila et al., 2013, 2012; Quesada et al., 2010) a llevar a cabo los controles necesarios para garantizar la captura completa de la información requerida para la evaluación del desempeño municipal en esta materia, asegurando así mejores resultados en la gestión adecuada de los recursos públicos.

\section{REFERENCIAS BIBLIOGRÁFICAS}

Arenas Valencia, W., Soto Mejía, J. A., \& Marino Rivera, O. (2004). La evaluación de los grupos de investigación según los indicadores de eficiencia de Colciencias versus su evaluación según el Análisis Envolvente de Datos- DEA. Scientia et Technica.

Asmild, M., Paradi, J. C., Aggarwall, V., \& Schaffnit, C. (2004). Combining DEA window analysis with the Malmquist index approach in a study of the Canadian banking industry. In Journal of Productivity Analysis (Vol. 21, pp. 67-89). http://doi.org/10.1023/B:PROD.0000012453.91326.ec

Banker, R. D., Charnes, A., \& Cooper, W. W. (1984). Some Models for Estimating Technical and Scale Inefficiencies in Data Envelopment Analysis. Management Science. http://doi.org/10.1287/ mnsc.30.9.1078

Barbosa Gómez, S. P. (2010). Evaluación de la eficiencia de las escuelas de la Universidad Industrial de Santander aplicando Análisis Envolvente de Datos (DEA). Universidad Industrial de Santander.

Bjurek, H., \& Hjalmarsson, L. (1995). Productivity in multiple output public service: A quadratic frontier function and Malmquist index approach. Journal of Public Economics, 56(3), 447-460. http:// doi.org/10.1016/0047-2727(94)01426-O

Cavadia, R., \& Maldonado, M. (2009). Análisis de la eficiencia del sector hotelero del distrito turístico de Cartaegena por medio del DEA. Universidad de Cartagena.

Caves, D. W., Christensen, L. R., \& Diewert, W. E. (1982). The economic theory of index numbers and the measurement of input, output, and productivity. Econometrica, 50(6), 1393-1414. http://doi. org/10.2307/1913388

Charnes, A., Cooper, W. W., \& Rhodes, E. (1978). Measuring the efficiency of decision making units. European Journal of Operational Research, 2(6), 429-444. http://doi.org/10.1016/03772217(78)90138-8

Chediak, F., \& Rodriguez, Y. (2011). La eficiencia relativa en cobertura educativa de los municipios del tolima, aplicando el análisis envolvente de datos -DEA- año 2009. Scientia et Technica Año XVII, (47), 44-48.

Coelli, T. J., \& Rao, D. S. P. (2005). Total factor productivity growth in agriculture: A Malmquist index analysis of 93 countries, 1980-2000. In Agricultural Economics (Vol. 32, pp. 115-134). http://doi.org/10.1111/ j.0169-5150.2004.00018.x

Coll, V., \& Blasco, O. M. (2006). Evaluación de la eficiencia mediante el análisis envolvente de datos: introducción a los modelos básicos. Eumed.net.

Färe, R., Grosskopf, S., Lindgren, B., \& Roos, P. (1989). Productivity Developments in Swedish Hospitals: A Malmquist Output Index Approach, 253-272. 
Fare, R., Grosskopf, S., Norris, M., \& Zhongyang Zhang. (1994). Productivity growth, technical progress, and efficiency change in industrialized countries. American Economic Review. http://doi. org/10.1111/j.1467-8268.2004.00089.x

Farrell, M. J. (1957). The Measurement of Productive Efficiency. Journal of the Royal Statistical Society. Series A (General). http://doi.org/10.1016/S0377-2217(01)00022-4

Gamarra, J. (2004). Eficiencia técnica relativa de la ganaderia doble propósito en la Costa Caribe. Documentos de Trabajo Sobre Economía Regional, 1-75.

Grifell-Tatjé, E., \& Lovell, C. A. K. (1995). A note on the Malmquist productivity index. Economics Letters, 47(2), 169-175. http://doi.org/10.1016/0165-1765(94)00497-P

Hjalmarsson, L., \& Veiderpass, A. (1992). Productivity in Swedish Electricity Retail Distribution. The Scandinavian Journal of Economics, 94, S193. http://doi.org/10.2307/3440259

Iregui B., A. M., Melo F, L., \& Ramos, J. (2006). Evaluación y análisis de eficiencia de la educación en Colombia. Banco de la República.

Kumar, S. (2006). Environmentally sensitive productivity growth: A global analysis using Malmquist-Luenberger index. Ecological Economics, 56(2), 280-293. http://doi.org/10.1016/j.ecolecon.2005.02.004

Liu, F. H. F., \& Wang, P. hsiang. (2008). DEA Malmquist productivity measure: Taiwanese semiconductor companies. International Journal of Production Economics, 112(1), 367-379. http://doi. org/10.1016/j.ijpe.2007.03.015

López Gómez, S. M., \& Rivera Monsalve, F. J. (2008). Determinación índice de eficiencia en educación pública, municipios del Departamento de Risaralda año 2005. Universidad Tecnológica de Pereira.

Lopez Munoz, O. M., \& Suarez Baquero, E. M. (2012). evaluacion de la eficiencia de las instituciones educativas oficiales de bucaramanga mediante el analisis envolvente de datos DEA. Universidad Industrial de Santander.

Malmquist, S. (1953). Index numbers and indifference surfaces. Trabajos de Estadistica, 4(2), 209-242. http://doi.org/10.1007/BF03006863

Marcelino Martinez, C. (2002). Productividad y eficiencia en la gestión pública del transporte de ferrocarriles: implicaciones de política económica. España.

Martín Bofarull, M. (2002). El sistema portuario español: regulación, entorno competitivo y resultados. una aplicación del DEA. Universitat Rovira I Virgili.

Maza Avila, F. J., Quesada Ibargüen, V. M., \& Vergara Schmalbach, J. C. (2013). Eficiencia y productividad de la calidad educativa en municipios del departamento de Bolívar-Colombia. Revista Entramado, 9(2), 28-39.

Maza Avila, F. J., Vergara Schmalbach, J. C., \& Navarro España, J. L. (2012). Eficiencia de la inversión en el régimen subsidiado en salud en Bolívar - Colombia. Investigaciones Andina, 14(24), 386-400.

MEN. (2009a). Organización del Sistema Educativo. Conceptos Generales de la educación preescolar, básica y media. Bogotá.

MEN. (2009b). Sistema General de Participaciones - Educación. 
Pérez González, M. del C., Maza Ávila, F. J., Blanco Canto, M., \& Jiménez García, M. (2016). Eficiencia y productividad de las políticas de empleo en la Eurozona. Revista de Ciencias Sociales (RCS), XXII(1), 11-25.

Quesada, V., Blanco, I., \& Maza, F. (2010). Análisis envolvente de datos aplicado a la cobertura educativa en el departamento de Bolívar - Colombia (2007-2008). Omnia, 16 No.3, 77-100.

Quesada Ibargüen, V. M., Blanco Hernández, I., \& Maza Avila, F. J. (2010). Análisis envolvente de datos aplicado a la cobertura educativa en el departamento de Bolívar - Colombia ( 2007-2008). Omnia, 16(3), 77-100.

Restrepo, M. I., \& Villegas R., J. G. (2007). Clasificación de grupos de investigación colombianos aplicando análisis envolvente de datos. Revista Facultad de Ingeniería, 42, 105-119.

Sanchez, M., Parra, G., \& Udi, S. (2014). Benchmarking de la sutentabilidad de empresas: una propuesta basada en el Análisis Envolvente de Datos. Trayectorias, 39, 19-35.

Soto Mejía, J. A., Vásquez Artunduaga, S., \& Villegas Flórez, J. A. (2009). Medición de la eficiencia en las instituciones educativas oficiales del municipio de Dosquebradas (Risaralda) 2007. Scientia Et Technica, 3(43), 1-5.

Torres Cardemas, J. C., \& Reynosa Carrero, A. C. (2011). Análisis económico de la eficiencia de los servicios de educación secundaria en Bogotá para los años 2005, 2006 y 2007. Universidad De La Salle.

\section{Para citaciones:}

Maza-Avila, F., Batista-Ochoa, I. \& Vergara-Schmalbach, J. C.(2017). La cobertura educativa en escuelas públicas del departamento de Bolívar (Colombia): Un análisis de eficiencia y productividad. Panorama Económico, 25, 4, pp. 559-576.

\section{AUTORES}

\section{Francisco Maza-Avila}

Profesor e Investigador del Programa de Administración Industrial de la Facultad de Ciencias Económicas de la Universidad de Cartagena (Colombia). Doctor en Ciencias Sociales y Jurídicas de la Universidad de Cádiz (España) y Magister en Desarrollo Territorial de la misma institución. Director del Grupo de Investigación en Estudios para el Desarrollo Regional de la Universidad de Cartagena

\section{Ivan Batista-Ochoa}

Administrador Industrial de la Universidad de Cartagena. Asistente de apoyo logístico en el Departamento de Investigaciones, Emprendimiento e Innovación de la Universidad Tecnológica de Bolívar (Colombia).

Juan Carlos Vergara-Schmalbach

Profesor e Investigador del Programa de Administración Industrial de la Facultad de Ciencias Económicas de la Universidad de Cartagena (Colombia). Candidato a Doctor en Ciencias Sociales (mención Gerencia) de la Universidad del Zulia (República Bolivariana de Venezuela). Director del Grupo de Investigación Métodos Cuantitativos de Gestión 